Castro, J.; Gabarró, J.; Serna, M. Refining the imprecise meaning of non-determinism in the Web by strategic games. A: International Conference on Computational Collective Intelligenge. "Computational Collective Intelligence, 11th International Conference, ICCCI 2019: Hendaye, France, September 4-6, 2019: proceedings, part I". Berlín: Springer, 2019, p. 566-578. The final authenticated version is available online at https://doi.org/10.1007/978-3-030-28377-3_47

\title{
Refining the imprecise meaning of non-determinism in the Web by strategic games ${ }^{\star}$
}

\author{
Jorge Castro $^{[0000-0002-1390-1313]}$, Joaquim Gabarro $^{[0000-0003-3771-2813]}$, Maria \\ Serna ${ }^{[0000-0001-9729-8648]}$ \\ Computer Science Department. Universitat Politècnica de Catalunya, Barcelona. \\ \{castro, gabarro, mjserna\}ecs.upc.edu
}

\begin{abstract}
Nowadays interactions with the World Wide Web are ubiquitous. Users interact through a number of steps consisting of site calls and handling results that can be automatized as orchestrations. Orchestration results have an inherent degree of uncertainty due to incomplete Web knowledge and orchestration semantics are characterized in terms of imprecise probabilistic choices. We consider two aspects in this imprecise semantic characterization. First, when local knowledge (even imprecise) of some part of the Web increases, this knowledge goes smoothly through the whole orchestration. We deal formally with this aspect introducing orchestration refinements. Second, we analyze refinement under uncertainty in the case of parallel composition. Uncertain knowledge is modeled by an uncertainty profile. Such profiles allow us to look at the uncertainty through a zero-sum game, called angel/daemon-game, or $\mathfrak{a} / \mathfrak{d}$-game. We propose to use the structure of the Nash equilibria to refine uncertainty. In this case the information improves not through cooperation but through the $\mathfrak{a}$ and $\mathfrak{d}$ competition.
\end{abstract}

Keywords. Web, orchestrations, refinement, non-deterministic choice, probabilistic and uncertain reasoning, $\mathfrak{a} / \mathfrak{d}$-games.

\section{Introduction}

Users interact with the Web through services. In our view an interaction involves a number of steps, each step consisting of some site or basic service calls and the handling of the results of the call. Often, this type of interactions can be automatized as orchestrations. In a real scenario where only a partial knowledge on the Web behaviour can be assumed, orchestration results have some uncertainty degree. The inherent uncertainty of an orchestration is reflected in the semantic characterization in terms of imprecise probabilistic choices proposed in [2].

We analyse how a better Web knowledge affects orchestration imprecise semantics. We introduce a partial order [1] on orchestration semantics and we show that, as expected, a decrease on Web uncertainty is transferred to a better (less imprecise) characterization.

\footnotetext{
* J. Castro was partially supported by MINECO and FEDER grant TIN2017- 89244-R and AGAUR grant 2017 SGR-856. J. Gabarro and M. Serna were partially supported by MINECO and FEDER grant TIN2017-86727-C2-1-R and AGAUR 2017 SGR 786. M. Serna was also supported by MINECO grant MDM-2014-044.
} 
Delays on delivering site call answers have a crucial role on the orchestration output. Frequently our knowledge about delays is imprecise. Even having tight bounds on time delays it is difficult to provide an orchestration assessment. A way to deal with this problem is to look at the uncertainty through an strategic situation with two antagonistic players the angel $\mathfrak{a}$ and the daemon $\mathfrak{d}[3]$. The angel $\mathfrak{a}$ tries to force the best possible scenario while the daemon $\mathfrak{d}$ tries to worsen it. This strategic approach provides an scenario in between the optimistic and the pessimistic ones. We apply this point of view and we show that it allows to refine orchestration semantics.

The paper is organized as follows. In Section 2, we introduce basic concepts concerning orchestrations and Web uncertainty. In particular, we introduce the notion of knowledge framework to deal with imprecise return times of site calls. In Section 3 , we introduce a partial order on orchestration semantics and we show a refinement result. In Section 4, we adapt uncertainty profiles and $\mathfrak{a} / \mathfrak{d}$-games to develop the idea of forget-refinement. In Section 5, we summarize the work and consider some possible extensions.

\section{Web under stress: imprecision}

An orchestration is a user-defined program that uses services on the Web. An orchestrator may utilize any service that is available on the Web. Although a concrete language is not necessary to present the problem under investigation, our results rely in a precise semantics characterization of the Orc language [13|11]. In particular, Orc will allow us to develop the interplay between non-determinism and imprecise probabilities in a mathematical setting. As we will see, the analysis of the Orc expressions strongly guide the mathematical approach.

In the Orc language services are modelled by sites having predefined semantics $\mathrm{A}$ site is silent if it does not publish any result. A site call publishes at most one response. An orchestration which composes a number of site calls into a complex computation can be represented by an Orc expression.

We only deal with orchestrations generating a finite number of results. Two Orc expressions $E$ and $F$ can be combined using the following operators [13]11]. The symmetric parallelism $E \mid F: E$ and $F$ are evaluated in parallel. $E \mid F$ publishes some interleaving of the streams published by $E$ and $F$. The asymmetric parallelism $E(x)<x<F: E$ and $F$ are evaluated in parallel. Some sub-expressions in $E$ may become blocked by a dependency on $x$. The first result published by $F$ is bound to $x$, the remainder of $F$ 's evaluation is terminated and the evaluation of the blocked residue of $E$ is resumed. Finally, the sequence $E>x>F(x)$ : $E$ is evaluated and, for each value $v$ published by $E$, an instance $F(v)$ is executed. Given an orchestration $E$ we denote by sites $(E)$ the set of sites in the definition of $E$. Information on delays is given by an evaluation function $\delta$ providing the return time of each orchestration site.

Example 1. In MaryNews orchestration sites $C N N$ and $B B C^{1}$ are called in parallel and the result of the first one to answer is emailed to Mary. This procedure can be described in Orc as: MaryNews $=$ Mary $(x)<x<$ TwoNews where TwoNews $=$

\footnotetext{
${ }^{1}$ A call to $C N N$ or $B B C$ can be interpreted as a call to https: / / edition. cnn. com/ or https://www.bbc.com/news
} 
$C N N \mid B B C$. Orchestration sites are $C N N$ and $B B C$ in TwoNews and site Mary $(x)$ providing an email service to Mary. Roughly, the information received by Mary depends on the response times (the delays) of $C N N$ and $B B C$, denoted as $\delta(C N N)$ and $\delta(B B C)$. Depending on the delays several cases can arise as we will see in the examples below.

In order to characterize ex-ante the execution of an orchestration $E$, we introduced in [5] the meaning or semantics of $E$, denoted by $\llbracket E \rrbracket$. When there is no information about return times but we know that orchestration results are $m_{1}, \ldots, m_{k}$, semantics $\llbracket E \rrbracket$ is given by the multiset $\left\lfloor m_{1}, \ldots, m_{k}\right\rfloor$ abstracting away any time order ${ }^{2}$, and we write $\llbracket E \rrbracket=\left\lfloor m_{1}, \ldots, m_{k}\right\rfloor$. As we will see later, a non-deterministic choice ${ }^{3}$ of multisets $M_{i}$ may be necessary to express the semantics of an orchestration. We write in this case $\llbracket E \rrbracket=\sqcap_{i} M_{i}$. First of all, we consider an example with no information about delays. When delay time $\delta$ is used to analyze semantics of $E$ we write $\llbracket E \rrbracket_{\delta}$.

Example 2. Let consider Example 1 under lack of information about delays. Suppose $\llbracket C N N \rrbracket=\lfloor\mathrm{cnn}\rfloor$ and $\llbracket B B C \rrbracket=\lfloor$ bbc $\rfloor$. Then $\llbracket T w o N e w s \rrbracket=\lfloor\mathrm{cnn}, \mathrm{bbc}\rfloor$. Assume that both delays are unknown, encoded as $\delta(C N N)=\perp$ and $\delta(B B C)=\perp$. Parameter $x$ in MaryNews $=\operatorname{Mary}(x)<x<$ TwoNews will get either cnn or bbc, in fact the first one to arrive. As we do not have any prior knowledge of the first arrival we assume $\left.\llbracket x \rrbracket_{\delta}=\lfloor\mathrm{cnn}\rfloor\right\rfloor \sqcap\lfloor\mathrm{bbc}\rfloor$, i. e. a non-deterministic choice of two small multisets. Then $\llbracket$ MaryNews $\rrbracket_{\delta}=\lfloor\lfloor$ mary_cnn $\rfloor \sqcap\lfloor$ mary_bbc $\rfloor$ pointing out that Mary gets an email with news provided by either $C N N$ or $B B C$.

Having full knowledge means that the delay function $\delta$ is known and it is defined on each orchestration site. We take from [9] the notation $P \triangleleft Q \triangleright R$. It should be read: $P$ if $Q$ else $R$.

Example 3. We assume true values $\delta(C N N)$ and $\delta(B B C)$ are known. Under the hypothesis $\delta(C N N) \neq \delta(B B C)$ we have

$$
\llbracket M a r y N e w s \rrbracket_{\delta}=\lfloor\text { mary_cnn }\rfloor \triangleleft \delta(C N N)<\delta(B B C) \triangleright\lfloor\text { mary_bbc }\rfloor .
$$

For function $\delta(C N N)=5$ and $\delta(B B C)=6, \llbracket x \rrbracket_{\delta}=\left\lfloor\lfloor\mathrm{cnn}\rfloor\right.$ and $\llbracket M a r y N e w s \rrbracket_{\delta}=$ $\lfloor$ mary_cnn $\rfloor$.

When (consistent) information increases the imprecision reduces. However, it is impossible to avoid completely the non-determinism.

Example 4. Assume that $\delta(C N N)$ and $\delta(B B C)$ could have the same value, a case that assuming discrete time is feasible. Here, a race condition give us

$$
\begin{aligned}
\llbracket M a r y N e w s \rrbracket_{\delta}= & \lfloor\lfloor\text { mary_cnn }\rfloor \sqcap\lfloor\text { mary_bbc }\rfloor \triangleleft \delta(C N N)=\delta(B B C) \triangleright \\
& (\lfloor\text { mary_cnn }\rfloor \triangleleft \delta(C N N)<\delta(B B C) \triangleright\lfloor\text { mary_bbc }\rfloor) .
\end{aligned}
$$

\footnotetext{
${ }^{2}$ Notations are taken from [12].

${ }^{3}$ We take from [10] the non-determinism $P \sqcap Q$ (called daemonic choice).
} 
When a site $S$ is under stress, information about delay is uncertain. Uncertainty refers here to response time, as a natural feature of network searches. As opposed to risk, we assume lack of data on distributions. To deal with this lack of probabilistic information, we adopt here a two antagonistic approach. When site $S$ is overloaded, the stress can be exerted by the angel $\mathfrak{a}$ or the daemon $\mathfrak{d}$ or both. We denote by $\delta_{\mathfrak{a}}(S), \delta_{\mathfrak{d}}(S)$ the corresponding level of stress. We assume that $\delta_{\mathfrak{d}}(S) \geq 0, \delta_{\mathfrak{a}}(S) \leq 0$. We denote by $S_{\mathfrak{d}}$ site $S$ under daemonic stress, in such a case $\delta\left(S_{\mathfrak{d}}\right)=\delta(S)+\delta_{\mathfrak{d}}(S)$. Similarly, $S_{\mathfrak{a}}$ denotes $S$ under angelic stress; here delay decreases to $\delta\left(S_{\mathfrak{a}}\right)=\delta(S)+\delta_{\mathfrak{a}}(S)$. It could happen that $S$ is under the joint action of $\mathfrak{a}$ and $\mathfrak{d}$ denoted as $S_{\mathfrak{a}, \mathfrak{d}}$ and $\delta\left(S_{\mathfrak{a}, \mathfrak{d}}\right)=$ $\delta(S)+\delta_{\mathfrak{a}}(S)+\delta_{\mathfrak{d}}(S)$.

Definition 1. A knowledge framework is a tuple $\mathcal{K}=\left\langle E, \delta, \delta_{\mathfrak{a}}, \delta_{\mathfrak{d}}\right\rangle$ where $\delta$ is the delay function and $\delta_{\mathfrak{a}}$ and $\delta_{\mathfrak{d}}$ provide the delay bounds under stress. Let $(a, d) \subseteq \operatorname{sites}(E) \times$ sites $(E)$ be a pair of site subsets under respectively $\mathfrak{a}$ stress (subset a) and $\mathfrak{d}$ stress (subset $d$ ). We evaluate $S[a, d]$, under risk conditions, as follows.

$$
S[a, d]=\left\{\begin{array}{l}
S \text { if } S \notin a \cup d \\
S_{\mathfrak{a}} \text { if } S \in a \backslash d \\
S_{\mathfrak{d}} \text { if } S \in d \backslash a \\
S_{\mathfrak{a}, \mathfrak{o}} \text { if } S \in a \cap d
\end{array}\right.
$$

We denote $E[a, d]$ the orchestration under stress where each $S \in \operatorname{sites}(E)$ has been replaced by $S[a, d]$.

When we want to emphasize $\mathcal{K}$, we write $S_{\mathcal{K}}[a, d]$ and $E_{\mathcal{K}}[a, d]$. The delay cost function $\mathcal{D}\left(E_{\mathcal{K}}[a, d]\right)$ is the delay of the first return based on $\delta\left(S_{\mathcal{K}}[a, d]\right)$. Next example borrows many ideas from a typical fuzzy approach.

Example 5. Consider the following knowledge framework $\mathcal{K}$ for MaryNews.

\begin{tabular}{|l|l|l|l||r|r|r|r|}
\hline & $\delta$ & $\delta_{\mathfrak{a}}$ & $\delta_{\mathfrak{l}}$ & & $\delta$ & $\delta_{\mathfrak{a}}$ & $\delta_{\mathfrak{l}}$ \\
\hline$C N N$ & 5 & -2 & 3 & $B B C$ & 6 & -4 & 2 \\
\hline
\end{tabular}

Take $(a, d)=(\{C N N, B B C\},\{\})$. Then TwoNews $_{\mathcal{K}}[a, d]=\left(C N N_{\mathfrak{a}} \mid B B C_{\mathfrak{a}}\right)$ and $\operatorname{MaryNews}_{\mathcal{K}}[a, d]=\operatorname{Mary}(x)<x<$ TwoNews $_{\mathcal{K}}[a, d]$. Then $\mathcal{D}\left(\right.$ TwoNews $\left._{\mathcal{K}}[a, d]\right)=$ $\min \{5-2,6-4\}=2$. As $B B C_{\mathfrak{a}}$ returns before than $C N N_{\mathfrak{a}}$ the result of the orchestration is $\llbracket$ MaryNews $_{\mathcal{K}}[a, d] \rrbracket=\lfloor$ mary_bbc $\rfloor$. Other results are also possible depending on $(a, d)$, for instance $\llbracket$ MaryNews $_{\mathcal{K}}[\{C N N\},\{\}] \rrbracket=\lfloor$ mary_cnn $\left.\rfloor\right\rfloor$.

\section{Imprecise probability and refinement}

A semantics for orchestrations where non-determinism is modeled with imprecise probabilities was proposed in [2]. We adapt this approach to deal with delays. Let $\Delta_{k}=$ $\left\{\left(p_{1}, \ldots, p_{k}\right) \mid \forall i: p_{i} \geq 0\right.$ and $\left.\sum_{i} p_{i}=1\right\}$ be a $k$ probabilistic space with $k-1$ degrees of freedom. 
Example 6. Let us revisit Example 2 where $\delta(C N N)=\delta(B B C)=\perp$. We encode $\llbracket x \rrbracket_{\delta}=\lfloor\lfloor\mathrm{cnn}\rfloor \sqcap\lfloor$ bbc $\rfloor$ through imprecise probabilities, ip for short, into

$$
\llbracket x \rrbracket_{i p}=\lfloor\mathrm{cnn}\rfloor @ \mu_{1} \rrbracket\lfloor\mathrm{bbc}\rfloor @ \mu_{2} .
$$

for some indefinite probability vector $\left(\mu_{1}, \mu_{2}\right)$ in $\Delta_{2}$. Last expressions show that semantics of parameter $x$ is the result of a probabilistic choice between two multisets with imprecise weights $\mu_{1}$ and $\mu_{2}$. Therefore,

$$
\llbracket \text { MaryNews } \rrbracket_{i p}=\lfloor\text { mary_cnn }\rfloor @ \mu_{1} \llbracket\lfloor\text { mary_bbc }\rfloor @ \mu_{2} .
$$

In the case we need to emphasize the set structure in the probabilistic choices we write

$$
\begin{aligned}
\llbracket x \rrbracket_{i p} & =\left\{\left\lfloor\lfloor\mathrm{cnn}\rfloor @ \mu_{1} \rrbracket\lfloor\mathrm{bbc}\rfloor @ \mu_{2} \mid\left(\mu_{1}, \mu_{2}\right) \in \Delta_{2}\right\}\right. \\
& =\left\{\left\lfloor\lfloor\mathrm{cnn}\rfloor @ \mu_{1} \rrbracket\lfloor\mathrm{bbc}\rfloor @ \mu_{2} \mid \mu_{1}, \mu_{2} \geq 0 \text { and } \mu_{1}+\mu_{2}=1\right\} .\right.
\end{aligned}
$$

Therefore, $\llbracket$ MaryNews $\rrbracket_{i p}$ is represented by

$$
\left\{\lfloor\text { mary_cnn }\rfloor @ \mu_{1} \llbracket\left\lfloor\lfloor\text { mary_bbc }\rfloor @ \mu_{2} \mid \mu_{1}, \mu_{2} \geq 0 \text { and } \mu_{1}+\mu_{2}=1\right\} .\right.
$$

We adapt from Theorem 3 in [2] the definition of multiset probabilistic choice to our setting. Given $\ell$ multisets $M_{1}, \ldots, M_{\ell}$ and a Cartesian product of probability spaces $\Delta=\Delta_{m_{1}} \times \cdots \times \Delta_{m_{k}}$, we introduce the imprecise probabilistic choice of multisets $M=\rrbracket_{1 \leq i \leq \ell} M_{i} @ P_{i}(\mu)$ where multiset $M_{i}$ is chosen with probability $P_{i}(\mu)$; here $\mu$ is any element of $\Delta$ and $P_{i}$ 's are arithmetic expressions on $\mu$ adding up one. Imprecision comes from the fact that value of $\mu$ is indefinite. In set notation

$$
M=\left\{\rrbracket_{1 \leq i \leq \ell} M_{i} @ P_{i}(\mu) \mid \mu \in \Delta \text { and } \sum_{1 \leq i \leq \ell} P_{i}(\mu)=1\right\} .
$$

We isolate the different probabilities arising at $M$ as

$$
\operatorname{prbs}(M)=\left\{\left(P_{1}(\mu), \ldots, P_{\ell}(\mu)\right) \mid \mu \in \Delta\right\} .
$$

We consider some examples.

Example 7. From Example 3, we have $\llbracket x \rrbracket_{\delta}=\lfloor\lfloor\mathrm{cnn}\rfloor=(\lfloor\lfloor\mathrm{cnn}\rfloor @ 1 \rrbracket\lfloor\mathrm{bbc}\rfloor\rfloor 0)$. Therefore $P_{1}=1, P_{2}=0$ and then prbs $\left(\llbracket x \rrbracket_{\delta}\right)=\{(1,0)\}$. From Example 6, we have $\operatorname{prbs}\left(\llbracket x \rrbracket_{i p}\right)=\left\{\left(\mu_{1}, \mu_{2}\right) \mid \mu_{1}+\mu_{2}=1\right\} \subseteq[0,1] \times[0,1]$. Also we have that $\operatorname{prbs}\left(\llbracket\right.$ MaryNews $\left.\rrbracket_{i p}\right)=\operatorname{prbs}\left(\llbracket x \rrbracket_{i p}\right)$. Now, consider another possible meaning (see Example 12 below for details).

$$
\left.\llbracket x \rrbracket_{\mathrm{rf}}=\lfloor\lfloor\mathrm{cnn}\rfloor\rfloor\left(\frac{8}{25}+\mu_{1} \frac{2}{25}\right) \rrbracket\lfloor\mathrm{bbc}\rfloor\right\rfloor\left(\frac{3}{5}+\mu_{2} \frac{2}{25}\right),
$$

where $\left(\mu_{1}, \mu_{2}\right)$ is an indefinite pair in $\Delta_{2}$. In this case $\mu=\left(\mu_{1}, \mu_{2}\right)$ and, writing

$$
P_{1}(\mu)=\frac{8}{25}+\mu_{1} \frac{2}{25}, P_{2}(\mu)=\frac{3}{5}+\mu_{2} \frac{2}{25},
$$


we get $\llbracket x \rrbracket_{\mathrm{rf}}=\left\lfloor\lfloor\mathrm{cnn}\rfloor @ P_{1}(\mu) \rrbracket\lfloor\mathrm{bbc}\rfloor @ P_{2}(\mu)\right.$, for indefinite $\mu \in \Delta_{2}$. Probabilities are

$$
\operatorname{prbs}\left(\llbracket x \rrbracket_{\text {rf }}\right)=\left\{\left(\frac{8}{25}+\mu_{1} \frac{2}{25}, \frac{3}{5}+\mu_{2} \frac{2}{25}\right) \mid \mu_{1}+\mu_{2}=1\right\} .
$$

Note that prbs $\left(\llbracket x \rrbracket_{\mathrm{rf}}\right) \subseteq[0.32,0.4] \times[0.4,0.6]$. Also

$$
\llbracket \operatorname{MaryNews} \rrbracket_{\mathrm{rf}}=\left\lfloor\lfloor\text { mary_cnn }\rfloor @ P_{1}(\mu) \rrbracket\lfloor\text { mary_bbc }\rfloor @ P_{2}(\mu)\right.
$$

and prbs $\left(\llbracket M a r y N e w s \rrbracket_{\mathrm{rf}}\right)=\operatorname{prbs}\left(\llbracket x \rrbracket_{\mathrm{rf}}\right)$.

In order to compare $\llbracket x \rrbracket_{i p}$ and $\llbracket x \rrbracket_{r f}$, we introduce refinements in the case of multiset probabilistic choice. This needs some careful considerations. For probability distributions, standard deviation is mostly accepted as a measure of being "more precise". Following [8], we consider a different approach.

Given $\ell$ multisets $\left(M_{1}, \ldots, M_{\ell}\right)$ and a Cartesian product of probability spaces $\Delta$ we would like to compare probabilistic multisets over the $M_{i}$ and $\Delta$. Given $\left(F_{1}(\mu), \ldots F_{\ell}(\mu)\right)$ and $\left(G_{1}\left(\mu^{\prime}\right), \ldots G_{\ell}\left(\mu^{\prime}\right)\right)$ for $\mu, \mu^{\prime} \in \Delta$ such that $\sum_{i} F_{i}(\mu)=\sum_{j} G_{j}(\mu)=1$, consider the probabilistic multisets

$$
M=\left\{\rrbracket_{1 \leq i \leq \ell} M_{i} @ F_{i}(\mu) \mid \mu \in \Delta\right\}, M^{\prime}=\left\{\rrbracket_{1 \leq j \leq \ell} M_{j} @ G_{j}\left(\mu^{\prime}\right) \mid \mu^{\prime} \in \Delta\right\} .
$$

The set of probability distributions are,

$$
\begin{aligned}
& \operatorname{prbs}(M)=\left\{\left(F_{1}(\mu), \ldots F_{\ell}(\mu)\right) \mid \mu \in \Delta\right\} \\
& \operatorname{prbs}\left(M^{\prime}\right)=\left\{\left(G_{1}\left(\mu^{\prime}\right), \ldots G_{\ell}\left(\mu^{\prime}\right)\right) \mid \mu^{\prime} \in \Delta\right\}
\end{aligned}
$$

We say that $M$ is more imprecise than $M^{\prime}$ (or $M^{\prime}$ is more precise than $M$ ), denoted as $M \sqsubseteq M^{\prime}$ if probabilities in $M^{\prime}$ are less spread than in $M$, formally iff prbs $\left(M^{\prime}\right) \subseteq$ prbs $(M)$. This approach makes sense because,

- any choice in $M^{\prime}$ is also a choice in $M$,

- moreover, both choices appear with the same probability distribution.

As in $M^{\prime}$ there are less possible choices than in $M$, uncertainty increases from $M^{\prime}$ to $M$. In this framework, increasing the imprecision means increasing the number of probabilistic choices. We summarize this point of view in the following definition.

Definition 2. Given $\ell$ multisets $M_{1}, \ldots, M_{\ell}$ and two multiset choices with imprecise weights $M=\rrbracket_{1 \leq i \leq \ell} M_{i} @ F_{i}(\mu)$ and $M^{\prime}=\rrbracket_{1 \leq i \leq \ell} M_{i} @ G_{i}\left(\mu^{\prime}\right)$ for, respectively, indefinite elements $\mu$ and $\mu^{\prime}$ in $\Delta$. We say that $M$ is more imprecise than $M^{\prime}$ (or $M^{\prime}$ is more precise than $M)$, denoted as $M \sqsubseteq M^{\prime}$ iff prbs $\left(M^{\prime}\right) \subseteq \operatorname{prbs}(M)$.

Example 8. Let us continue with Example 7. As prbs $\left(\llbracket x \rrbracket_{\delta}\right) \subseteq \operatorname{prbs}\left(\llbracket x \rrbracket_{i p}\right)$, according to Definition 2, we have $\llbracket x \rrbracket_{i p} \sqsubseteq \llbracket x \rrbracket_{\delta}$. As prbs $\left(\llbracket x \rrbracket_{\text {rf }}\right) \subseteq \operatorname{prbs}\left(\llbracket x \rrbracket_{i p}\right)$, we have $\llbracket x \rrbracket_{i p} \sqsubseteq \llbracket x \rrbracket_{\mathrm{rf}}$. Observe that $\llbracket x \rrbracket_{\mathrm{rf}}$ and $\llbracket x \rrbracket_{\delta}$ are not comparable, as prbs $\left(\llbracket x \rrbracket_{\delta}\right) \nsubseteq$ $\operatorname{prbs}\left(\llbracket x \rrbracket_{\mathrm{rf}}\right)$ and prbs $\left(\llbracket x \rrbracket_{\mathrm{rf}}\right) \nsubseteq \operatorname{prbs}\left(\llbracket x \rrbracket_{\delta}\right)$.

Theorem 1. Let $F$ be a sub-orchestration of $E=E(F)$. Suppose that our knowledge $\llbracket F \rrbracket$ improves into $\llbracket F^{\prime} \rrbracket$, i.e., $\llbracket F \rrbracket \sqsubseteq \llbracket F^{\prime} \rrbracket$. Then, the increase of knowledge goes smoothly through the whole orchestration; $\llbracket E(F) \rrbracket \sqsubseteq \llbracket E\left(F^{\prime}\right) \rrbracket$. 


\begin{tabular}{|c|c|c|c|c|c|}
\hline \multicolumn{4}{|c|}{ d } & \multicolumn{2}{|c|}{$\mathfrak{d}$} \\
\hline & $\{C N N$ & $3 B C\}$ & & $\{C N N\}$ & $\{B B C\}$ \\
\hline$\{C N N\}$ & 6 & 3 & $\{C N N\}$ & $C N N_{\mathfrak{a}, \mathfrak{d}} \sqcap B B C$ & $C N N_{\mathfrak{a}}$ \\
\hline$\{B B C\}$ & 2 & 4 & $\{B B C\}$ & $B B C_{\mathfrak{a}}$ & $B B C_{\mathfrak{a}, \mathfrak{d}}$ \\
\hline
\end{tabular}

Fig. 1. Take $\mathcal{U}=\left\langle\mathcal{K}\right.$, sites $(T w o N e w s)$, sites $($ TwoNews $\left.), 1,1, \Delta\left(E_{\mathcal{K}}[a, d]\right)\right\rangle$. The left table corresponds to $\Gamma(\mathcal{U})$ and the right table to the values of the indicator function.

Proof (Sketch). We proceed by structural induction showing that refinement is monotone through Orc operators. As an illustration, let us consider the parallel composition case. Take $E=F \mid G$ where

$$
\llbracket F \rrbracket=\rrbracket_{1 \leq i \leq \ell} M_{i} @ P_{i}(\mu), \llbracket G \rrbracket=\rrbracket_{1 \leq j \leq m} N_{j} @ Q_{j}(\gamma),
$$

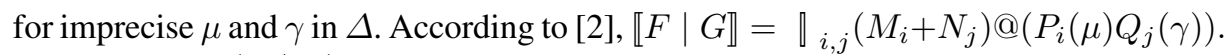
We write $\operatorname{prbs}(F \mid G)$ when $\ell=m=2$,

$$
\left\{\left(P_{1}(\mu) Q_{1}(\gamma), P_{1}(\mu) Q_{2}(\gamma), F_{2}(\mu) G_{1}(\gamma), F_{2}(\mu) G_{2}(\gamma)\right) \mid \mu, \gamma \in \Delta\right\} .
$$

Suppose $\llbracket G \rrbracket \sqsubseteq \llbracket H \rrbracket$ with $\llbracket H \rrbracket=\rrbracket_{1<j<m} N_{j} @ R_{j}(\tau)$ for imprecise $\tau$ in $\Delta$, so $\operatorname{prbs}(\llbracket H \rrbracket) \subseteq \operatorname{prbs}(\llbracket G \rrbracket)$. For $m=2$ this inclusion can be written as,

$$
\left\{( R _ { 1 } ( \tau ) , R _ { 2 } ( \tau ) | \tau \in \Delta \} \subseteq \left\{\left(Q_{1}(\gamma), Q_{2}(\gamma) \mid \gamma \in \Delta\right\} .\right.\right.
$$

For each value $P_{i}(\mu)$, it holds that $\left\{\left(P_{i}(\mu) R_{1}(\tau), P_{i}(\mu) R_{2}(\tau) \mid \tau \in \Delta\right\}\right.$ is contained into $\left\{\left(P_{i}(\mu) Q_{1}(\gamma), P_{i}(\mu) G_{2}(\gamma) \mid \gamma \in \Delta\right\}\right.$. Therefore prbs $(\llbracket F \mid H \rrbracket) \subseteq \operatorname{prbs}(\llbracket F \mid$ $G \rrbracket)$ and $\llbracket F|G \rrbracket \sqsubseteq \llbracket F| H \rrbracket$ holds. With the definitions given in [2], we prove similar results for the asymmetric parallelism and sequence operators.

Example 9. From Example 7 we get prbs $\left(\llbracket M a r y N e w s \rrbracket_{i p}\right)=\operatorname{prbs}\left(\llbracket x \rrbracket_{i p}\right)$ and also $\operatorname{prbs}\left(\llbracket\right.$ MaryNews $\left.\rrbracket_{\mathrm{rf}}\right)=\operatorname{prbs}\left(\llbracket x \rrbracket_{\mathrm{rf}}\right)$. Therefore $\llbracket x \rrbracket_{i p} \sqsubseteq \llbracket x \rrbracket_{\mathrm{rf}}$ translates directly into $\llbracket$ MaryNews $\rrbracket_{i p} \sqsubseteq \llbracket$ MaryNews $\rrbracket_{r f}$.

\section{Uncertainty profiles and refinement}

Stressed orchestrations can deliver different results depending on the location of the stress, see for instance Example 5 above. If we bound the spread of the stress but it is not possible to locate it, what can be said about the delay? To give an answer to this question we adapt uncertainty profiles to this framework [3].

A uncertainty profile $\mathcal{U}$ takes a closer look to a knowledge profile $\mathcal{K}=\left\langle E, \delta, \delta_{\mathfrak{a}}, \delta_{\mathfrak{d}}\right\rangle$ providing bounds to the spread and intensity of the stress. The stress effects are measured through a cost (or utility) function. Formally $\mathcal{U}=\left\langle\mathcal{K}, \mathcal{A}, \mathcal{D}, b_{\mathfrak{a}}, b_{\mathfrak{d}}, \mathcal{D}\left(E_{\mathcal{K}}[a, d]\right)\right\rangle$ where agents (or players) $\mathfrak{a}$ and $\mathfrak{d}$ have the capability to act on subsets of sites $\mathcal{A}$ and $\mathcal{D}$ 
of the orchestration $E$ from $\mathcal{K}$. Parameters $b_{\mathfrak{a}}$ and $b_{\mathfrak{d}}$ give the number of sites that $\mathfrak{a}$ or $\mathfrak{d}$ can stress. The effects of the joint interaction of $\mathfrak{a}$ and $\mathfrak{d}$ is measured by $\mathcal{D}\left(E_{\mathcal{K}}[a, d]\right)$.

As in [3], we associate to $\mathcal{U}$ a zero-sum $\mathfrak{a} / \mathfrak{d}$-game $\Gamma(\mathcal{U})=\left\langle A_{\mathfrak{a}}, A_{\mathfrak{d}}, \mathcal{D}\left(E_{\mathcal{K}}[a, d]\right)\right\rangle$ with two antagonistic players the angel $\mathfrak{a}$ and the daemon $\mathfrak{d}$. The actions of $\mathfrak{a}$ and $\mathfrak{d}$ are given by the sets $A_{\mathfrak{a}}=\left\{a \subseteq \mathcal{A} \mid \# a=b_{\mathfrak{a}}\right\}$ and $A_{\mathfrak{d}}=\left\{d \subseteq \mathcal{D} \mid \# d=b_{\mathfrak{d}}\right\}$. The delay $\mathcal{D}\left(E_{\mathcal{K}}[a, d]\right)$ becomes a cost function. Angel $\mathfrak{a}$ tries to minimize the delay and $\mathfrak{d}$ to maximize it. The set of strategy profiles is $A_{\mathfrak{a}} \times A_{\mathfrak{d}}$. Choices for $\mathfrak{a}$ and $\mathfrak{d}$ can be defined probabilistically. Mixed strategies for $\mathfrak{a}$ and $\mathfrak{d}$ are probability distributions $\alpha: A_{\mathfrak{a}} \rightarrow[0,1]$ and $\beta: A_{\mathfrak{d}} \rightarrow[0,1]$ respectively. Delay of the mixed strategy $(\alpha, \beta)$ is $\mathcal{D}\left(E_{\mathcal{K}}[\alpha, \beta]\right)=\sum_{(a, d) \in A_{\mathfrak{a}} \times A_{\mathfrak{d}}} \alpha(a) \mathcal{D}\left(E_{\mathcal{K}}[a, d]\right) \beta(d)$. Let $\Delta_{\mathfrak{a}}$ and $\Delta_{\mathfrak{d}}$ denote the set of mixed strategies for $\mathfrak{a}$ and $\mathfrak{d}$, respectively. A pure strategy profile $(a, d)$ is a special case of mixed strategy $(\alpha, \beta)$ in which $\alpha(a)=1$ and $\beta(d)=1$. A mixed strategy profile $(\alpha, \beta)$ is a Nash equilibrium if, for any $\alpha^{\prime} \in \Delta_{\mathfrak{a}}$, it holds $\mathcal{D}\left(E_{\mathcal{K}}[\alpha, \beta]\right) \leq \mathcal{D}\left(E_{\mathcal{K}}\left[\alpha^{\prime}, \beta\right]\right)$ ( $\mathfrak{a}$ tries to minimize the delay as possible; going out of the Nash cannot reduce delay) and, for any $\beta^{\prime} \in \Delta_{\mathfrak{d}}$, it holds $\mathcal{D}\left(E_{\mathcal{K}}[\alpha, \beta]\right) \geq \mathcal{D}\left(E_{\mathcal{K}}\left[\alpha, \beta^{\prime}\right]\right)$ ( $\mathfrak{d}$ tries to maximize the delay; going out of the Nash cannot increase delay). A pure Nash equilibrium, PNE, is a Nash equilibrium $(a, d)$ with pure strategies.

It holds that all Nash equilibrium (pure and mixed) of a zero-sum game $\Gamma$ have the same value $\nu(\Gamma)$ corresponding to the cost of the row player [14]. For an $\mathfrak{a} / \mathfrak{d}$ game $\Gamma(\mathcal{U})$ we have:

$$
\nu(\Gamma(\mathcal{U}))=\min _{\alpha \in \Delta_{\mathfrak{a}}} \max _{\beta \in \Delta_{\mathfrak{o}}} \mathcal{D}\left(E_{\mathcal{K}}[\alpha, \beta]\right)=\max _{\beta \in \Delta_{\mathfrak{o}}} \min _{\alpha \in \Delta_{\mathfrak{a}}} \mathcal{D}\left(E_{\mathcal{K}}[\alpha, \beta]\right) .
$$

This observation is important because it allows us to associate a delay to an uncertain situation looking at it as a zero-sum game. We take this value as the result of the strategic approach to uncertainty.

Definition 3. The delay associated to $\mathcal{U}$ is $\mathcal{D}(\mathcal{U})=\nu(\Gamma(\mathcal{U}))$.

When $(\alpha, \beta)$ is a Nash equilibrium it holds $\mathcal{D}(\mathcal{U})=\mathcal{D}\left(E_{\mathcal{K}}[\alpha, \beta]\right)$.

Example 10. We consider for orchestration TwoNews $=C N N \mid B B C$ the knowledge profile $\mathcal{K}$ in Example 5 . We examine the uncertainty profile $\mathcal{U}$ defined as

$$
\left\langle\mathcal{K}, \operatorname{sites}(\text { TwoNews }), \operatorname{sites}(\text { TwoNews }), 1,1, \mathcal{D}\left(E_{\mathcal{K}}[a, d]\right)\right\rangle,
$$

where both sites can be stressed but the angel (respectively, the daemon) affects only to one site. Along this example we shorten $\mathcal{D}\left(E_{\mathcal{K}}[a, d]\right)$ as $\mathcal{D}(a, d)$. Actions of $\mathfrak{a}$ in $\Gamma(\mathcal{U})$ are given by $A_{\mathfrak{a}}=\{a \subseteq \operatorname{sites}($ TwoNews $) \mid \# a=1\}=\{\{C N N\},\{B B C\}\}$. As $A_{\mathfrak{d}}=A_{\mathfrak{a}}$, the set of strategy profiles $A_{\mathfrak{a}} \times A_{\mathfrak{d}}$ is

$$
\{(\{C N N\},\{C N N\}),(\{C N N\},\{B B C\}),(\{B B C\},\{C N N\}),(\{B B C\},\{B B C\})\} .
$$

It holds

$$
\mathcal{D}(\{C N N\},\{C N N\})=\min \left\{\delta(C N N)+\delta_{\mathfrak{a}}(C N N)+\delta_{\mathfrak{d}}(C N N), \delta(B B C)\right\}=6 .
$$

Other cases are computed similarly. The $\mathfrak{a} / \mathfrak{d}$-game is shown on the left table in the Fig.1. A strategy giving the Nash equilibrium for $\mathfrak{a}, \alpha=(\alpha(\{C N N\}), \alpha(\{B B C\}))$, 
can be found solving the equation $\mathcal{D}(\alpha,\{C N N\})=\mathcal{D}(\alpha,\{B B C\})$. Similarly, to get $\beta$ for $\mathfrak{d}$ we solve $\mathcal{D}(\{C N N\}, \beta)=\mathcal{D}(\{B B C\}, \beta)$. Then $\alpha=(2 / 5,3 / 5)$ and $\beta=(1 / 5,4 / 5)$. Thus, the expected return time on the first output of TwoNews in equilibrium is

$$
\mathcal{D}(\mathcal{U})=\frac{2}{5} \times 6 \times \frac{1}{5}+\frac{2}{5} \times 3 \times \frac{4}{5}+\frac{3}{5} \times 2 \times \frac{1}{5}+\frac{3}{5} \times 4 \times \frac{4}{5}=\frac{18}{5} .
$$

We are interested in modelling how the $\mathfrak{a} / \mathfrak{d}$-games are able to refine the imprecise knowledge on asymmetric parallelism. Consider $E(x)<x<F$ where $F$ is a parallel composition of sites $S_{1}|\cdots| S_{k}$. Assume $\llbracket S_{i} \rrbracket=\left\lfloor\left\lfloor\mathbf{s}_{i}\right\rfloor\right\rfloor$, then $\llbracket F \rrbracket=\left\lfloor\mathbf{s}_{1}, \ldots, \mathbf{s}_{k}\right\rfloor$, and, with no delay time information $(\delta=\perp)$, we can only infer that parameter $x$ will hold any of the values in $\llbracket F \rrbracket$. So, $\llbracket x \rrbracket=\left\lfloor\mathbf{s}_{1}\right\rfloor \sqcap \cdots \sqcap\left\lfloor\mathbf{s}_{k}\right\rfloor$. The imprecise probabilistic model gives

$$
\left.\llbracket x \rrbracket_{i p}=\left\lfloor\mathbf{s}_{1}\right\rfloor @ \mu_{1} \rrbracket \cdots \rrbracket\left\lfloor\mathbf{s}_{k}\right\rfloor\right\rfloor \mu_{k},
$$

for an indefinite probability vector $\left(\mu_{1}, \ldots, \mu_{k}\right)$ in $\Delta_{k}$, and so

$$
\llbracket E(x)<x<F \rrbracket_{i p}=\rrbracket_{1 \leq i \leq k} \llbracket E\left(\mathbf{s}_{i}\right) \rrbracket @ \mu_{i} .
$$

In order to provide an expression for the refinement of $\llbracket x \rrbracket_{i p}$ through $\mathfrak{a} / \mathfrak{d}$-games, we introduce some additional concepts.

Definition 4. Let $\mathcal{U}=\left\langle\mathcal{K}, \mathcal{A}, \mathcal{D}, b_{\mathfrak{a}}, b_{\mathfrak{d}}, \mathcal{D}\left(E_{\mathcal{K}}[a, d]\right)\right\rangle$, where $\mathcal{K}=\left\langle F, \delta, \delta_{\mathfrak{a}}, \delta_{\mathfrak{d}}\right\rangle$ and $F$ is a parallel composition of sites $S_{1}|\cdots| S_{k}$. For each strategy profile $(a, d)$ we consider $F[a, d]=T_{1}|\cdots| T_{k}$ where $T_{\ell}=S_{\ell}[a, d]$, for $1 \leq \ell \leq k$. The indicator function of strategy profile $(a, d)$ in $\Gamma(\mathcal{U})$ is the set consisting of all $S_{\ell}$ sites such that $\delta\left(T_{\ell}\right)$ is minimum among $\left\{\delta\left(T_{1}\right), \ldots, \delta\left(T_{k}\right)\right\}$. Formally,

$$
I_{\mathcal{U}}(a, d)=\left\{S_{\ell} \mid \delta\left(T_{\ell}\right)=\mathcal{D}(F[a, d])\right\} .
$$

Proposition 1. Let $(\alpha, \beta)$ be a Nash equilibrium in $\Gamma(\mathcal{U})$. For any $S \in I_{\mathcal{U}}(a, d)$ it holds:

$$
\mathcal{D}(\mathcal{U})=\sum_{a, d} \alpha(a) \delta(S[a, d]) \beta(d) .
$$

Proof. By definition, $\mathcal{D}(\mathcal{U})=\sum_{a, d} \alpha(a) \mathcal{D}(F[a, d]) \beta(d)$. As $S \in I_{\mathcal{U}}(a, d)$, it holds $\delta(S[a, d])=\mathcal{D}(F[a, d])$ and we get the result.

Let us introduce refinements $\llbracket x \rrbracket_{\mathcal{U}}$, provided by $\Gamma(\mathcal{U})$ game, on the $\llbracket x \rrbracket_{i p}$ semantics.

Example 11. We continue with the Example 10 emphasizing the stress suffered by the sites, see the right table in Fig. 1. This table points out the sites giving minimum return time in $(C N N \mid B B C)[a, d]$. For instance, when $(a, d)=(\{C N N\},\{C N N\})$, the table value is a non-deterministic choice between $C N N_{\mathfrak{a}, \mathfrak{d}}$ and $B B C$ representing that $I_{\mathcal{U}}(C N N, C N N)$ is $\{C N N, B B C\}$. The following refinement of $\llbracket x \rrbracket_{i p}$ can be introduced from a Nash equlibrium and the indicators functions of $\Gamma(\mathcal{U})$. First consider a version where the $\mathfrak{a} / \mathfrak{d}$ stress over the sites is explicitly added when needed to improve readability, 
$\left(\left(\llbracket C N N_{\mathfrak{a}, \mathfrak{d}} \rrbracket \sqcap \llbracket B B C \rrbracket\right) @ \frac{2}{5} \times \frac{1}{5} \rrbracket \llbracket C N N_{\mathfrak{a}} \rrbracket @ \frac{2}{5} \times \frac{4}{5} \rrbracket \llbracket B B C_{\mathfrak{a}} \rrbracket @ \frac{3}{5} \times \frac{1}{5} \rrbracket \llbracket B B C_{\mathfrak{a}, \mathfrak{d}} \rrbracket @ \frac{3}{5} \times \frac{4}{5}\right)$.

Finally $\llbracket x \rrbracket_{i p}$ is

$$
\left((\llbracket C N N \rrbracket \sqcap \llbracket B B C \rrbracket) @ \frac{2}{5} \times \frac{1}{5} \rrbracket \llbracket C N N \rrbracket @ \frac{2}{5} \times \frac{4}{5} \rrbracket \llbracket B B C \rrbracket @ \frac{3}{5} \times \frac{1}{5} \rrbracket \llbracket B B C \rrbracket @ \frac{3}{5} \times \frac{4}{5}\right) .
$$

Note that in this case $\mathcal{D}(\mathcal{U})$ is $18 / 5$.

The formal definition of the $\mathcal{U}$ refinements that fixes stress exerted by $\mathfrak{a}$ and $\mathfrak{d}$ over the sites according to weigthed strategy profiles in $\Gamma(\mathcal{U})$ in a Nash equilibrium is as follows

Definition 5. The $\mathcal{U}$ refinements of $\llbracket x \rrbracket_{i p}$ is given by

$$
\llbracket x \rrbracket_{\mathcal{U}}=\rrbracket_{a, d}\left(\sqcap_{S \in I_{\mathcal{U}}(a, d)} \llbracket S \rrbracket\right) @ \alpha(a) \times \beta(d),
$$

where $(\alpha, \beta)$ is a Nash equilibrium of $\Gamma(\mathcal{U})$.

Example 12. For the Nash equilibrium in Example 10 , the $\mathcal{U}$ refinement for $\llbracket x \rrbracket_{i p}$ is

$$
\begin{aligned}
\llbracket x \rrbracket_{\mathcal{U}} & =(\llbracket C N N \rrbracket \sqcap \llbracket B B C \rrbracket) @ \frac{2}{5} \times \frac{1}{5} \rrbracket \llbracket C N N \rrbracket @ \frac{2}{5} \times \frac{4}{5} \rrbracket \llbracket B B C \rrbracket @ \frac{3}{5} \times \frac{1}{5} \rrbracket \llbracket B B C \rrbracket @ \frac{3}{5} \times \frac{4}{5} \\
& =\left(\lfloor\mathrm{cnn} \rrbracket \sqcap\lfloor\mathrm{bbc}\rfloor) @ \frac{2}{5} \times \frac{1}{5} \rrbracket\left\lfloor\mathrm{cnn} \rrbracket @ \frac{2}{5} \times \frac{4}{5} \rrbracket\lfloor\mathrm{bbc}\rfloor @ \frac{3}{5} .\right.\right.
\end{aligned}
$$

Replacing the daemonic choice $\sqcap$ by imprecise probabilities and regrouping, we have

$$
\lfloor\mathrm{cnn}\rfloor @\left(\frac{2}{5} \times \frac{4}{5}+\mu_{1} \times \frac{2}{5} \times \frac{1}{5}\right) \rrbracket\lfloor\mathrm{bbc}\rfloor @\left(\frac{3}{5}+\mu_{2} \times \frac{2}{5} \times \frac{1}{5}\right),
$$

for some indefinite probability vector $\left(\mu_{1}, \mu_{2}\right)$ in $\Delta_{2}$. Using $\mathcal{U}$, from Example 6 , we get the meaning into

$$
\llbracket x \rrbracket_{\mathcal{U}}=\lfloor\mathrm{cnn}\rfloor @\left(\frac{8}{25}+\mu_{1} \frac{2}{25}\right) \rrbracket\lfloor\mathrm{bbc}\rfloor @\left(\frac{3}{5}+\mu_{2} \frac{2}{25}\right),
$$

where probability vector $\left(\mu_{1}, \mu_{2}\right)$ is indefinite. Then, we have the following meaning for MaryNews, $\llbracket$ MaryNews $\rrbracket_{\mathcal{U}}$

$$
\lfloor\text { mary_cnn }\rfloor @\left(\frac{8}{25}+\mu_{1} \frac{2}{25}\right) \rrbracket\lfloor\text { mary_bbc }\rfloor @\left(\frac{3}{5}+\mu_{2} \frac{2}{25}\right) .
$$

As $\llbracket x \rrbracket_{i p}$ is described by any probability distribution, prbs $\left(\llbracket x \rrbracket_{\mathcal{U}}\right) \subseteq \operatorname{prbs}\left(\llbracket x \rrbracket_{i p}\right)$. Moreover, if we assume that the environment behaves as predicted by $\mathcal{U}$, a more precise behaviour can be announced. 
Theorem 2. Given $E(x)<x<F$ where $F=\left(S_{1}|\cdots| S_{k}\right)$ and an uncertainty profile $\mathcal{U}$ on $F$ we have $\llbracket x \rrbracket_{i p} \sqsubseteq \llbracket x \rrbracket_{\mathcal{U}}$ and $\llbracket E(x)<x<F \rrbracket_{i p} \sqsubseteq \llbracket E(x)<x<F \rrbracket_{\mathcal{U}}$. Assuming that $F$ behaves as predicted by $\Gamma(\mathcal{U})$, the arrival times to $x$ of the different possible values follow the $\llbracket x \rrbracket_{\mathcal{U}}$ distribution. Moreover, assuming that the execution of $E$ is triggered by $x, \mathbb{E}(\mathcal{D}(E(x)<x<F))=\mathcal{D}(E)+\mathcal{D}(\mathcal{U})$.

In this way, using induction and additional rules for more complex composition, we can associate a meaning to any $E$ under uncertainty profile $\mathcal{U}, \llbracket E \rrbracket_{\mathcal{U}}$ which is a refinement on $\llbracket E \rrbracket_{i p}$.

\section{Conclusions}

Although cooperation is the usual model to improve situations, in real scenarios it is more likely to find some sort of competition. We have shown a way to use the competition between agents, $\mathfrak{a}$ and $\mathfrak{d}$, to improve our knowledge of the environment. Our approach allows to consider more realistic scenarios and provides an additional analysis tool to support the decisions of the system managers. Here, we have continued the work in [2] considering the relation between imprecise probability semantics and the $\mathfrak{a} / \mathfrak{d}$-approach. In particular, we have shown that the structure of Nash equilibria due to $\mathfrak{a} / \mathfrak{d}$-games can be used to refine the imprecise meaning of $E(x)<x<F$ which is the unique composition rule that inserts additional non-determinism in the behaviour of an orchestration. Our results also show a monotonic behaviour of imprecise meaning versus the level of knowledge. We are working towards understanding better the framework, in particular, monotonicity properties with respect to different uncertainty profiles in general orchestrations.

Nash equilibria and the value of the $\mathfrak{a} / \mathfrak{d}$-game is a natural solution for refinement, however finding Nash equilibria in $\mathfrak{a} / \mathfrak{d}$-games can be computationally difficult, in fact it is an EXP-complete problem [4]. As $\mathfrak{a} / \mathfrak{d}$-games are zero-sum games, it might also be possible to find the game values through iterative methods [16]. Furthermore, the approach through mixed strategies, appearing in the Nash equilibria, seems also to suggest the possibility to develop algorithms based Monte Carlo techniques, that could be more efficient. It will be of interest to explore these approaches.

It will also be of interest to analyse whether the $\mathfrak{a} / \mathfrak{d}$-refinement applies to other settings in particular to short-term economic systems like the IS-LM or IS-MP models [6] or other decision support models. Finally it could be interesting to apply this approach to more applied problems like real politics [7] or climate change [15].

\section{References}

1. Allison, L.: A Practical Introduction to Denotational Semantics. Cambridge (1986)

2. Castro, J., Gabarro, J., Serna, M.: Web apps and imprecise probabilitites. In: Medina, J., Ojeda-Aciego, M., Galdeano, J.L.V., Pelta, D.A., Cabrera, I.P., Bouchon-Meunier, B., Yager, R.R. (eds.) Information Processing and Management of Uncertainty in Knowledge-Based Systems. Theory and Foundations - 17th International Conference, IPMU 2018, Cádiz, Spain, June 11-15, 2018, Proceedings, Part II. Communications in Computer and Information Science, vol. 854, pp. 226-238. Springer (2018). https://doi.org/10.1007/978-3-31991476-3_19, https://doi.org/10.1007/978-3-319-91476-3_19 
3. Gabarro, J., Serna, M., Stewart, A.: Analysing web-orchestrations under stress using uncertainty profiles. The Computer Journal 57(11), 1591-1615 (2014)

4. Gabarro, J., Garcia, A., Serna, M.: Computational aspects of uncertainty profiles and angeldaemon games. Theory Comput. Syst. 54(1), 83-110 (2014)

5. Gabarro, J., Leon-Gaixas, S., Serna, M.: The computational complexity of QoS measures for orchestrations. Journal of Combinatorial Optimization 34(4), 1265-1301 (2017)

6. Gabarro, J., Serna, M.: Uncertainty in basic short-term macroeconomic models with angel-daemon games. IJDATS 9(4), 314-330 (2017). https://doi.org/10.1504/IJDATS.2017.10009422, https://doi.org/10.1504/ IJDATS.2017.10009422

7. García-Sanz, M.D., Llamazares, I., Manrique, M.A.: Ideal and real party positions in the 2015-2016 spanish general elections. In: IPMU 2018, Part III. Communications in Computer and Information Science, vol. 855, pp. 52-62. Springer (2018)

8. He, J., Seidel, K., McIver, A.: Probabilistic models for the guarded command language. Sci. Comput. Program. 28(2-3), 171-192 (1997). https://doi.org/10.1016/S0167-6423(96)000196, https://doi.org/10.1016/S0167-6423(96)00019-6

9. Hoare, C.A.R.: A couple of novelties in the propositional calculus. Math. Log. Q. 31(912), 173-178 (1985). https://doi.org/10.1002/malq.19850310905, https://doi.org/ $10.1002 / \mathrm{malq} .19850310905$

10. Hoare, C.: Communicating Sequential Processes. Prentice-Hall, London (1985)

11. Kitchin, D., Quark, A., Cook, W., Misra, J.: The Orc programming language. In: Lee, D., Lopes, A., Poetzsch-Heffter, A. (eds.) Proceedings of FMOODS/FORTE 2009. Lecture Notes in Computer Science, vol. 5522. Springer (2009)

12. McIver, A., Morgan, C.C.: Abstraction, Refinement and Proof for Probabilistic Systems. Springer, New York (2005)

13. Misra, J., Cook, W.: Computation orchestration: A basis for wide-area computing. Software and Systems Modeling 6(1), 83-110 (2007)

14. von Neumann, J., Morgenstern, O.: Theory of games and economic behavior, 60th Anniversary Commemorative Edition. Princeton University Press, Princeton and Oxford (1953)

15. Nordhaus, W.: Climate Casino: Risk, Uncertainty, and Economics for a Warming World. Yale (2013)

16. Robinson, J.: An iterative method of solving a game. Annals of Mathematics, Second Series 54(2), 296-301 (1951) 